\title{
Frailty in the perioperative setting
}

\author{
Authors: Jugdeep K Dhesi, ${ }^{\mathrm{A}}$ Nicholas P Lees ${ }^{\mathrm{B}}$ and Judith SL Partridge ${ }^{\mathrm{C}}$
}

The frailty syndrome is defined as a decrease in physiological reserve across multiple organ systems leading to increased vulnerability to external stressors. Studies across surgical subspecialties and in emergency and elective settings have identified frailty as an independent predictor of adverse postoperative clinician-reported, patient-reported and process-related outcomes. Although frailty is not specific to the older population, it is associated with ageing and therefore is increasingly observed in the ageing surgical population. Identifying frailty early in the perioperative pathway affords the opportunity to assess risk, modify the syndrome, inform shared decision making and plan the surgical pathway. Multiple tools to screen and diagnose frailty exist with limited appraisal of clinometric properties. A pragmatic approach to these tools is advocated with a future focus on collaborative approaches to modify the syndrome using multicomponent methodology such as comprehensive geriatric assessment and adapt the pathway to the needs of the frail surgical patient.

KEYWORDS: Perioperative medicine, frailty, older surgical patients, comprehensive geriatric assessment and optimisation (CGA), postoperative complications

\section{Introduction}

Frailty is defined as a decrease in physiological reserve across multiple organ systems leading to increased vulnerability to even seemingly minor external stressors. This renders the frail individual at risk of poor resolution of homeostasis after a stressor event such that the threshold from independence to dependence is often crossed. ${ }^{1}$ For example, a frail person who develops a minor

Authors: ${ }^{A}$ consultant geriatrician, perioperative medicine for older people undergoing surgery (POPS) team, Guy's and St Thomas' NHS Foundation Trust, London, UK, honorary reader, Faculty of Life Sciences and Medicine, King's College London, London, UK and honorary associate professor, Division of Surgery \& Interventional Science, University College London, London, UK; ${ }^{\mathrm{B}}$ consultant general and colorectal surgeon, Salford Royal NHS Foundation Trust, Salford, UK; ' Consultant geriatrician, perioperative medicine for older people undergoing surgery (POPS) team, Guy's and St Thomas' NHS Foundation Trust, London, UK and honorary senior lecturer, Faculty of Life Sciences and Medicine, King's College London, London, UK infection is more likely to require external assistance with daily activities in comparison to a non-frail person who may continue to work in the context of a similar illness. In the surgical setting, such an acquired reduction in resilience is associated with more frequent postoperative complications, a longer length of hospital stay, higher mortality rates at 30 days and 12 months after surgery, and a greater chance of dependent living at hospital discharge. ${ }^{2,3}$ The association between frailty and increased risk of adverse postoperative clinician-reported, patient-reported and process-related outcomes has now been reported across all major surgical specialties, emergency and elective settings, and following critical care admission. ${ }^{4}$

Perioperative medicine is being established to provide optimal preoperative, intraoperative and postoperative care for all patients, but with a particular focus on those at high risk of adverse postoperative outcomes. The high-risk group is defined as surgical patients with an aggregate 90 -day mortality rate greater than $1 / 20 .^{5,6}$ These high-risk patients are predominantly those with age related physiological changes, accumulation of multimorbidity and geriatric syndromes including frailty. With the advent of this new speciality, perioperative medicine, it is no surprise that there has been a focus on identifying patients with frailty in order to modify the perioperative pathway and achieve improved outcomes for individual patients. ${ }^{6}$

\section{Models of frailty}

Two models of frailty have been described. The first of these, the frailty phenotype was developed through secondary analysis of data from the cardiovascular health study. ${ }^{7}$ The frailty phenotype proposes five variables (unintentional weight loss, self-reported exhaustion, low energy expenditure, slow gait speed and weak grip strength) and describes individuals with three or more of these variables as frail, those with one or two factors present as pre-frail and those with none of the variables as 'robust'. The frailty index or deficit accumulation model of frailty was derived from the Canadian study of health and ageing using 92 variables, including symptoms, signs, existing diagnoses and biochemical markers. ${ }^{8}$ The index uses a binary count of the absence or presence of each variable in an individual and thus calculates the number of accumulated deficits from the total possible deficit count, giving a result on a continuous scale from 0 (least frail) to 1 (most frail). The more deficits, the frailer the individual and the higher the risk of institutionalisation and death. In this original description of the frailty index, by the time the index reached about 0.67 , further deficit accumulation seemed impossible and death resulted. The index has been replicated using different sets of variables 
producing similar results provided the variables collected represent multiple domains.

Since the initial conceptualisation of frailty, there has been a recognition of the overlap between frailty and other geriatric syndromes. Fried described the relationship between frailty, comorbidity and disability in 2004 and, more recently, the shared characteristics between frailty, sarcopenia and cachexia particularly in terms of pathogenesis and clinical phenotype have been described. ${ }^{9,10}$ In addition, frailty should be considered in the context of wider determinants; modifiable life-course conditions including socioeconomic standing. ${ }^{11}$ These are closely linked to the concept of resilience, defined as the ability to adapt to or overcome stress or trauma. The World Health Organization's World report on ageing and health refers to intrinsic capacity as the composite of all physical and mental capacities that an individual can draw on to promote resilience and minimise negative health outcomes. ${ }^{12}$ Understanding and managing frailty in all clinical settings requires an understanding of these overlapping concepts and syndromes in order to deliver personalised medicine supported by robust clinical pathways grounded in socioeconomic, cultural and environmental conditions.

\section{Frailty in surgical patients}

Future projections predict that by 2030 , one-fifth of surgical procedures will be conducted in patients aged over 75 years. ${ }^{5}$ By the nature of surgical pathology, which is often degenerative (eg osteoarthritis), neoplastic (eg bladder cancer) or metabolic (eg vascular disease), it is unsurprising that the surgical population is ageing. While frailty is associated with ageing, it not exclusively observed in older people, nor are all older people frail. Numerous studies have described the prevalence of frailty with an observed variation in rates reported across surgical specialties. In those undergoing elective orthopaedic surgery, $23 \%$ were frail in comparison to emergency hip fracture surgery where $53 \%$ of patients were defined as frail. ${ }^{13,14}$ Considering cancer surgery, studies report a prevalence of $25 \%$ of patients undergoing elective cystectomy as being frail with a similarly high prevalence of frailty in emergency general surgical patients of $39 \%$ where the underlying pathology is often neoplastic. ${ }^{15,16}$ In vascular surgery, aortic aneurysms and peripheral arterial disease increase with age, with an estimated $52 \%$ of elective vascular patients being frail. ${ }^{17}$

\section{Identifying frailty in the perioperative setting}

Given that frailty is common with significant perioperative implications but is not exclusive to or universal in older people, accurately identifying frailty in the context of other overlapping conditions and syndromes is vital. While comprehensive geriatric assessment (CGA) and optimisation remains the gold standard method for screening, diagnosing and managing frailty, it can be time consuming and requires specialist skills. This has resulted in the development of numerous frailty tools designed to be applicable across clinical settings and deliverable by nonspecialists. These tools vary from single surrogate markers of frailty (eg gait velocity), simple infographic tools (eg clinical frailty scale (CFS)), scales or scores (eg Edmonton frailty scale or electronic frailty index), biomarkers (eg interleukin 6), disease specific scores (eg comprehensive assessment of frailty) or surgery specific scores (eg FORECAST) ${ }^{18-24}$ Furthermore, the opportunistic evaluation of related syndromes, such as sarcopenia, using cross sectional imaging has increased in popularity with suggestions that this may represent a surrogate marker for frailty. ${ }^{25}$ Such an approach is at odds with the definition of sarcopenia which is low muscle quantity or quality combined with low muscle strength and fails to recognise the differences between sarcopenia and frailty as discrete clinical syndromes. While the brevity and ease of surrogate markers is attractive, the use of these tools risks losing fidelity to the multidomain nature of the frailty definition and models.

A systematic review from 2016 examining frailty assessment instruments concluded that, despite the availability of numerous tools, there was limited evidence of discriminant validity, construct validity or reliability. Furthermore, the majority of tools were being used to predict adverse outcomes as opposed to aid with clinical decision making or provide an interventional target. ${ }^{26}$ In the perioperative setting, a similar systematic review suggested that despite the recognition that frailty is an independent risk factor for adverse outcomes, the question about the best clinical tool for assessing frailty remains unanswered. ${ }^{27}$ Challenges exist in accurately measuring frailty in the perioperative setting, particularly in expedited or urgent surgery. Disentangling the acute pathology from underlying frailty status can be difficult and may rely on collateral history and discussion with primary care. In the absence of consensus regarding which frailty tool should be used to either screen for, or diagnose, frailty in the perioperative setting, many clinicians have opted for a pragmatic approach choosing CFS. This screening tool can be used by non-specialists in the elective and emergency surgical setting and has been shown to be strongly associated with 30 - and 90-day mortality, risk of complications and length of intensive care unit and overall hospital stay after emergency laparotomy. Indeed, using CFS, even patients who are assessed as being vulnerable or pre-frail rather than frail, have outcomes that mark them out as high-risk. The CFS has now been included in the National Emergency Laparotomy Audit. 2,28

\section{Managing frailty in the perioperative pathway}

Identifying and managing frailty in the perioperative setting depends on the acuity of presentation; elective or emergency. In the elective setting, early screening and diagnosis of frailty is advocated. The benefit of frailty assessment at the start of the pathway include informing risk assessment, shared decision making and potential modification of the syndrome well in advance of potential surgery. An accurate diagnosis of frailty coupled with a knowledge of the perioperative implications of frailty on morbidity and mortality can promote informed discussion of the potential benefits, risks, alternatives to surgery or options if nothing is done. In such a situation, some patients may decide, together with healthcare professionals, not to undertake surgical management electing instead for conservative measures. In other cases, seemingly highrisk patients may work with healthcare teams to modify the frailty syndrome, thus altering the perioperative risk profile, allowing surgery to occur and improving postoperative outcomes. Furthermore, there is frequently more than one surgical option. For example, a patient considering surgery for rectal cancer may have options that include local resection, radical resection with a stoma and radical resection with restoration of bowel continuity. Each has different oncological benefits, perioperative risks and quality-of-life outcomes. Incorporating frailty assessment, preoperative optimisation, multidisciplinary shared decision making and targeted perioperative interventions means that some frail patients can still benefit from surgery who may have otherwise been considered too high-risk. 
Table 1. A multidomain approach to modifying the frailty syndrome in the perioperative setting. Adapted from Dhesi JK, Partridge JSL, Moppett IK. Anaesthesia for the older person. In: Thompson JP, Wiles MD, Moppett IK (eds), Smith and Aitkenhead's textbook of anaesthesia, 7th edn. Elsevier, 2019.

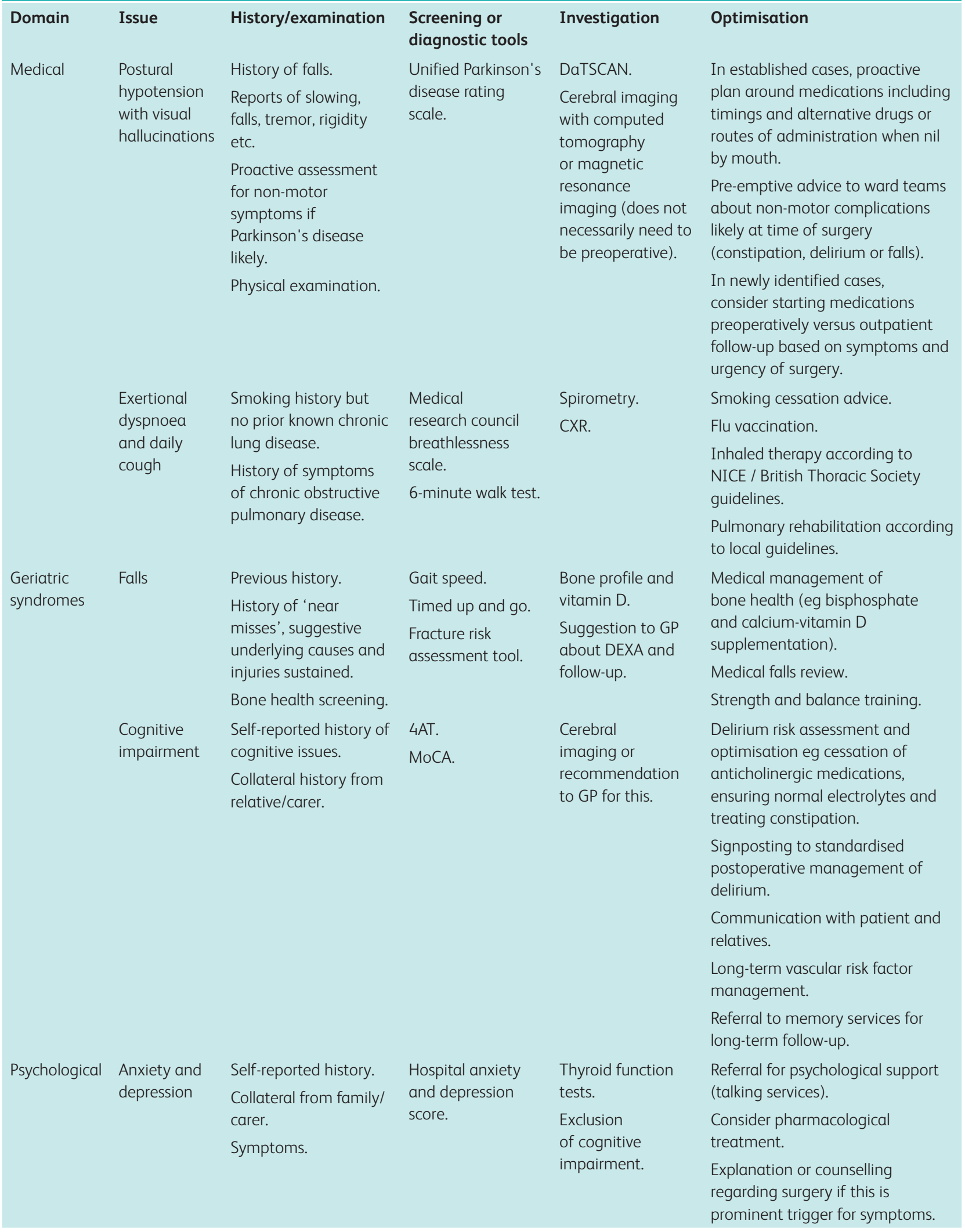


Table 1. (Continued).

\begin{tabular}{|c|c|c|c|c|c|}
\hline Domain & Issue & History/examination & $\begin{array}{l}\text { Screening or } \\
\text { diagnostic tools }\end{array}$ & Investigation & Optimisation \\
\hline \multirow{8}{*}{$\begin{array}{l}\text { Functional } \\
\text { and social }\end{array}$} & \multirow{5}{*}{$\begin{array}{l}\text { Functional } \\
\text { dependency }\end{array}$} & Self-reported concerns. & Barthel. & \multirow{5}{*}{$\begin{array}{l}\text { Physical } \\
\text { examination and } \\
\text { investigation of } \\
\text { pathology causing } \\
\text { disability eg } \\
\text { proximal myopathy } \\
\text { secondary } \\
\text { to vitamin D } \\
\text { deficiency. } \\
\text { Prescribe analgesia } \\
\text { for osteoarthritis. }\end{array}$} & Preoperative physiotherapy. \\
\hline & & $\begin{array}{l}\text { Collateral from family/ } \\
\text { carer. }\end{array}$ & \multirow{4}{*}{$\begin{array}{l}\text { Nottingham } \\
\text { extended activities } \\
\text { of daily living. }\end{array}$} & & $\begin{array}{l}\text { Occupational therapy intervention } \\
\text { (eg home adaptations). }\end{array}$ \\
\hline & & \multirow[t]{3}{*}{$\begin{array}{l}\text { Assessment of } \\
\text { underlying cause. }\end{array}$} & & & $\begin{array}{l}\text { Social worker intervention to } \\
\text { proactively identify barriers to } \\
\text { discharge. }\end{array}$ \\
\hline & & & & & $\begin{array}{l}\text { Proactive communication } \\
\text { regarding anticipated length of }\end{array}$ \\
\hline & & & & & $\begin{array}{l}\text { stay and access to rehabilitation or } \\
\text { care at discharge. }\end{array}$ \\
\hline & \multirow{3}{*}{$\begin{array}{l}\text { Non- } \\
\text { adherence } \\
\text { to prescribed } \\
\text { medications }\end{array}$} & $\begin{array}{l}\text { Self or family reported } \\
\text { concerns. }\end{array}$ & \multirow[t]{3}{*}{ STOPP/START. } & \multirow{3}{*}{$\begin{array}{l}\text { Assessment of } \\
\text { cognition and } \\
\text { understanding of } \\
\text { medications. }\end{array}$} & \multirow{3}{*}{$\begin{array}{l}\text { Liaising with community } \\
\text { pharmacist to assist with dosette } \\
\text { box and with care services or } \\
\text { telecare to prompt medication. }\end{array}$} \\
\hline & & $\begin{array}{l}\text { Clinical evidence of } \\
\text { non-adherence. }\end{array}$ & & & \\
\hline & & $\begin{array}{l}\text { Assessment of } \\
\text { understanding of } \\
\text { medications. }\end{array}$ & & & \\
\hline
\end{tabular}

$4 A T$ = four ' $A$ 's test; $C X R=$ chest $X$-ray; DaTscan = dopamine transporter single photon emission computed tomography; $D E X A$ = dual-energy $X$-ray absorptiometry; $\mathrm{GP}=$ general practitioner; MoCA = Montreal cognitive assessment; NICE = National Institute for Health and Care Excellence; START = screening tool to alert doctors to right treatments; STOPP = screening tool of older people's potentially inappropriate prescriptions.

In the emergency setting, the same principles apply but the emphasis shifts away from modifying the patients' risk profile instead to adapting the pathway of care. For example, high-risk frail patients undergoing emergency laparotomy will be managed by consultantlevel staff with planned level 3 care. Early discussions with patients and their families regarding ceilings of care may also be triggered by the recognition that the patient is frail with acknowledged adverse outcomes, avoiding the futility of surgery in some and the futility of escalating interventions after complications in others. ${ }^{6}$

While screening for frailty has gained traction in perioperative pathways, the interpretation of frailty tool results requires a skilled workforce. In keeping with the perioperative agenda, this requires a collaborative approach between surgeons, anaesthetics and those skilled in the management of frailty and multimorbidity. Such an approach should firstly focus on potential modifiers of the frailty syndrome at an individual patient level and secondly on modifying the perioperative pathway to achieve optimal clinicianreported, patient-reported and process-related outcomes.

\section{Modifying frailty in the perioperative setting}

No evidence-based single frailty modifier exists. Studies have examined pharmacological agents, exercise, nutritional supplementation and multicomponent interventions with limited success to date. Limitations to this body of work result from a lack of explicit frailty measures such that in many cases the findings are extrapolated from populations known to have a high prevalence of frailty, for example care home residents, hip fracture patients, sarcopenic subjects or those with pressure ulcers, but without frailty being explicitly defined or identified. Despite these acknowledged issues, no pharmacological interventions are currently supported by the literature, although there is support for the positive effect of angiotensin converting enzyme inhibitor (ACEi) medications on skeletal muscle function, testosterone on muscle strength and vitamin $D$ on neuromuscular functioning. Side effects, in the case of testosterone, and lack of convincing efficacy have limited the widespread use of these medications, although research into the use of ACEi as a therapeutic agent in those with sarcopenia is currently ongoing. ${ }^{29}$

Similarly, although the use of nutritional supplementation to slow or reverse the weight loss commonly associated with the frailty syndrome may seem therapeutically attractive, this has not yet been supported in research studies. Exercise intuitively seems sensible in a group known to be largely sedentary, with slow gait velocity, and is known to have positive physiological effects on the brain, endocrine system, immune system, and skeletal muscle. ${ }^{1}$ However, research examining the impact of exercise on modifying frailty is mixed, with suggestion that the most frail patients gain the least from this intervention. ${ }^{30}$ This does not preclude clinicians from recommending exercise programmes for other indications and positive results may emerge in future trials.

Translation of this scant evidence on frailty modification into the preoperative setting is even more problematic. While the national appetite for prehabilitation exercise programmes in older, frail, surgical populations has been considerable, to date there is no evidence linking these with improved postoperative outcomes. This leaves researchers and clinicians aware that frailty has an adverse impact on outcomes in older surgical populations, able to identify the syndrome using various tools but unable to effectively treat frailty with a single intervention or modifier evidenced to have benefit. In this situation the potential for multicomponent interventions to modify aspects of the frailty syndrome appear attractive. The established multicomponent intervention CGA has been shown to have benefit on morbidity and mortality in older frailer patients in other clinical areas.

\section{Comprehensive geriatric assessment and optimisation}

CGA and optimisation is an established method for evaluating and managing older patients in various clinical settings. It involves a multidomain, interdisciplinary assessment aiming to describe both 
known pathology and previously undiagnosed conditions together with evaluating functional, psychological and social status. This multidomain assessment prompts the formulation of a short- and longer-term investigation and management plan for all issues identified (Table 1). In the context of frailty in the perioperative setting, addressing a multisystem condition through a multidomain intervention has face validity and is increasingly supported by the perioperative literature. ${ }^{31,32}$ These studies provide evidence to support the hypothesis that utilising preoperative CGA can improve postoperative outcome in older surgical populations; hip fracture, orthopaedic elective surgery, elective vascular surgery and colorectal surgery. However, these studies have not directly examined the impact of CGA specifically on the frailty syndrome in the perioperative period.

\section{Discussion}

The surgical population is ageing and frailty is increasingly observed. With the recognition that this syndrome has an adverse impact on postoperative outcome, new approaches to perioperative medicine are necessary. The focus of future research and implementation science should be three-fold. First, achieving consensus on which frailty tool to use for screening and diagnosis in emergency and elective surgical settings rather than developing yet more frailty tools is paramount. Second, the case for frailty as a predictor of adverse postoperative outcomes is now well made and does not require further evaluation. Instead, research should investigate both multicomponent interventions and single pharmacological modifiers of the frailty syndrome in the perioperative setting. Third, these research findings should be translated to routine clinical care through the development of collaborative perioperative pathways and evaluated using implementation science methodology. .

\section{References}

1 Clegg A, Young J, Iliffe S et al. Frailty in elderly people. Lancet 2013;381:752-62.

2 Parmar KL, Law J, Carter B et al. Frailty in Older patients undergoing emergency laparotomy: results from the uk observational emergency laparotomy and frailty (ELF) study. Ann Surg 2019:[Epub ahead of print].

3 Muscedere J, Waters B, Varambally A et al. The impact of frailty on intensive care unit outcomes: a systematic review and metaanalysis. Intensive Care Med 2017:43:1105-22.

4 Lin HS, Watts JN, Peel NM et al. Frailty and post-operative outcomes in older surgical patients: a systematic review. BMC Geriat 2016:16:157

5 Fowler A, Abbott T, Prowle ] et al. Age of patients undergoing surgery. Br J Surg 2019;106:1012-8.

6 Lees NP, Peden C], Dhesi JK et al. The high-risk general surgery patient: Raising the standard. London: Royal College of Surgeons of England, 2018.

7 Fried LP, Tangen CM, Walston ] et al. Frailty in older adults: evidence for a phenotype. J Gerontol A Biol Sci Med Sci 2001;56:M146-56.

8 Jones D, Song X, Mitnitski A et al. Evaluation of a frailty index based on a comprehensive geriatric assessment in a population based study of elderly Canadians. Aging Clin Exp Res 2005;17:465-71.

9 Fried LP, Ferrucci L, Darer ] et al. Untangling the concepts of disability, frailty, and comorbidity: implications for improved targeting and care. J Gerontol A Biol Sci Med Sci 2004;59:255-63.

10 Partridge JS, Harari D, Dhesi JK. Frailty in the older surgical patient: a review. Age Ageing 2012;41:142-7.

11 Hale M, Shah S, Clegg A. Frailty, inequality and resilience. Clin Med 2019;19:219-23.
12 World Health Organization. World report on ageing and health. Geneva: WHO, 2015.

13 van de Ree CLP, Landers MJF, Kruithof $\mathrm{N}$ et al. Effect of frailty on quality of life in elderly patients after hip fracture: a longitudinal study. BMJ Open 2019;9:e025941.

14 Johnson RL, Abdel MP, Frank RD et al. Impact of Frailty on Outcomes After Primary and Revision Total Hip Arthroplasty. J Arthroplasty 2019;34:56-64.e5.

15 Khan $\mathrm{M}$, Jehan $\mathrm{F}$, Zeeshan $\mathrm{M}$ et al. Failure to rescue after emergency general surgery in geriatric patients: does frailty matter? J Surg Res 2019;233:397-402.

16 Chappidi MR, Kates M, Patel HD et al. Frailty as a marker of adverse outcomes in patients with bladder cancer undergoing radical cystectomy. Urol Oncol 2016;34:256.e1-6.

17 Partridge JS, Fuller M, Harari D et al. Frailty and poor functional status are common in arterial vascular surgical patients and affect postoperative outcomes. Int J Surg 2015;18:57-63.

18 Afilalo J, Eisenberg MJ, Morin JF et al. Gait speed as an incremental predictor of mortality and major morbidity in elderly patients undergoing cardiac surgery. J Am Coll Cardiol 2010;56:1668-76.

19 Rockwood K, Song X, Macknight C et al. A global clinical measure of fitness and frailty in elderly people. CMAJ 2005;173:489-95.

20 Rolfson DB, Majumdar SR, Tsuyuki RT et al. Validity and reliability of the Edmonton Frail Scale. Age Ageing 2006;35:526-9.

21 Clegg A, Bates C, Young ] et al. Development and validation of an electronic frailty index using routine primary care electronic health record data. Age Ageing 2016;45:353-60.

22 Hubbard RE, Woodhouse KW. Frailty, inflammation and the elderly. Biogerontology 2010;11:635-41.

23 Sundermann S, Dademasch A, Praetorius ] et al. Comprehensive assessment of frailty for elderly high-risk patients undergoing cardiac surgery. Eur ] Cardiothorac Surg 2011;39:33-7.

24 Kobe AR, Meyer A, Elmubarak $\mathrm{H}$ et al. Frailty assessed by the FORECAST is a valid tool to predict short-term outcome after transcatheter aortic valve replacement. Innovations (Phila) 2016;11:407-13.

25 Bentov I, Kaplan SJ, Pham TN et al. Frailty assessment: from clinical to radiological tools. $\mathrm{Br}$ ] Anaesth 2019;123:37-50.

26 Buta BJ, Walston JD, Godino JG et al. Frailty assessment instruments: systematic characterization of the uses and contexts of highly-cited instruments. Ageing Res Rev 2016;26:53-61.

27 Buigues C, Juarros-Folgado P, Fernandez-Garrido J et al. Frailty syndrome and pre-operative risk evaluation: a systematic review. Arch Gerontol Geriatr 2015;61:309-21.

28 The National Emergency Laparotomy Project Team. Fourth patient report of the National Emergency Laparotomy Audit. London: Royal College of Anaesthetists, 2018.

29 Band MM, Sumukadas D, Struthers AD et al. Leucine and ACE inhibitors as therapies for sarcopenia (LACE trial): study protocol for a randomised controlled trial. Trials 2018;19:6.

30 Hubbard RE, Fallah N, Searle SD et al. Impact of exercise in community-dwelling older adults. PLoS One 2009:4:e6174.

31 Partridge JS, Harari D, Martin FC et al. Randomized clinical trial of comprehensive geriatric assessment and optimization in vascular surgery. Br J Surg 2017;104:679-87.

32 Eamer G, Taheri A, Chen SS et al. Comprehensive geriatric assessment for older people admitted to a surgical service. Cochrane Database Syst Rev 2018;1:CD012485.

Address for correspondence: Prof Jugdeep K Dhesi, perioperative medicine for older people undergoing surgery (POPS) team, Ground Floor, Bermondsey Wing, Guy's Hospital, Great Maze Pond, London SE1 9RT, UK.

Email: jugdeep.dhesi@gstt.nhs.uk 